\title{
Targeting Hypoxic Tumor Microenvironment in Pancreatic Cancer: An Emerging Innovative Concept
}

ISSN: 2637-773X

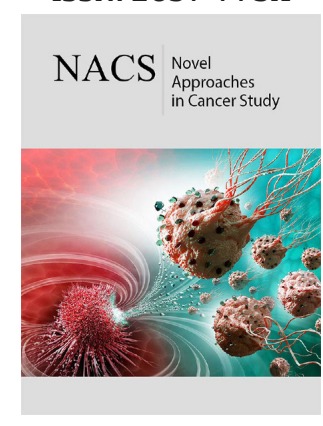

*Corresponding authors: Rinku Majumder, Department of Biochemistry \& Molecular Biology, USA. email: rmajum@lsuhsc.edu Samarpan Majumder, Department of Genetics, USA. e.mail: smaju1@lsuhsc.edu

Submission: 漹 September 07, 2021

Published: 梅 September 21, 2021

Volume 6 - Issue 3

How to cite this article: Rinku Majumder*, Tanusree Sengupta,Samarpan Majumder*. Targeting Hypoxic Tumor Microenvironment in Pancreatic Cancer: An Emerging Innovative Concept. Nov Appro in Can Study. 6(3). NACS.000636. 2021.

DOI: 10.31031/NACS.2021.06.000636

Copyright@ Rinku Majumder, Samarpan Majumder, This article is distributed under the terms of the Creative Commons Attribution 4.0 International License, which permits unrestricted use and redistribution provided that the original author and source are credited.

\author{
Rinku Majumder ${ }^{1 *}$, Tanusree Sengupta ${ }^{2}$ and Samarpan Majumder ${ }^{3 *}$ \\ ${ }^{1}$ Department of Biochemistry \& Molecular Biology, LSU Health Sciences Center, USA \\ ${ }^{2}$ Department of Chemistry, Sri Sivasubramaniya Nadar College of Engineering, Chennai, India \\ ${ }^{3}$ Department of Genetics, LSU Health Sciences Center, USA
}

\begin{abstract}
In spite of rapid progress in translational cancer research and advent of a few treatment regimens that revolutionized patient care in most of the tumors, any meaningful progress in the treatment of pancreatic cancer (PC) remains elusive. Lack of definitive early symptoms, absence of reliable biomarker and failure to understand PC biology combined with a complex PC tumor microenvironment (TME) slowed progress of therapeutics development to treat PC. One of the areas of PC biology that has been gaining a lot of attention of late is the extent of tumor hypoxia or lack of oxygen that is associated with PC progression. The goal of this mini review is to emphasize the importance of hypoxia in PC. Our improving understanding of hypoxia and how to make hypoxia amenable to targeted therapies further opens up new possibility to manage PC progression. We are focusing on a novel finding where a natural anticoagulant, protein S (PS) shows promise to control PC.
\end{abstract}

Keywords: Tumor microenvironment; Hypoxia; Protein S

Abbreviations: PC: Pancreatic Cancer; TME: Tumor Microenvironment; PS: Protein S

\section{Introduction}

Pancreatic ductal adenocarcinoma (PDAC) represents 90\% of all PC cases [1] and almost $100 \%$ of PDAC contains KRAS mutation [2]. However, therapeutic targeting of KRAS remains far from reality despite of our best efforts. While we made great strides in the treatment of many different solid tumors with immunotherapies, single-agent immunotherapies have failed to show significant clinical responses in PC [3-5]. Importantly, hypoxia is also associated with many different cancers, including PC [6,7]. This begets the possibility of targeting hypoxia as an "Achilles heel" in PC TME and control PC progression.

\section{Discussion}

According to NCI, in 2021, there will be an estimated 60431 new cases of PC and an estimated 48220 people will die from it, placing PC as the $3^{\text {rd }}$ largest case of cancer related deaths in US. Severe hypoxic regions are hallmarks of PC with a median tissue partial oxygen pressure $\left(\mathrm{pO}_{2}\right)$ of $0-5.3 \mathrm{mmHg}(0-0.7 \%)$ compared to the adjacent normal pancreas $\mathrm{pO}_{2}$ at 24.3-92.7mmHg (3.2-12.3\%) [8,9]. The presence of hypoxic areas within PC spur tumor progression and exacerbates poor clinical outcome in PC patients. Hypoxia is considered as one of the independent prognostic factors for PC [9]. PC induces a hypoxic tumor environment that drives the production of HIF $1 \alpha$ and stabilizes it $[9,10]$. Pilli et al. [11] have made the novel observation that protein S (PS), a natural human plasma protein that participates in coagulation control and has multiple receptor-mediated effects [12] has activity on in vitro models of pancreatic cancer (PC) cells. PS is a 75-kDa vitamin K-dependent glycoprotein synthesized by hepatocytes, endothelial cells, and megakaryocytes and is involved in many physiological processes; hemostasis, apoptosis, and inflammation [13]. The plasma concentration of PS is reportedly reduced during the progression of PDAC [14]. Pilli et al. [11] also demonstrated that expressing PS in PC cell lines reduces their aggressiveness. The investigators made a compelling case to supplement PS to control PC. Pilli et al. [15] have previously shown that PS deficiency augments the hypoxic niche by stabilizing HIF1 $\alpha$ and elevates thrombotic risk 
in obese mice. The authors demonstrated downregulation of HIF1 $\alpha$ by PS overexpression in HepG2 cell line [15]. Importantly, cancerassociated thrombosis (CAT) is a significant risk factor in PC, but little is known about its mechanistic basis $[16,17]$. Iodice et al. [18] inferred from a number of population studies that patients with venous thromboembolism (VTE) have a six-fold higher risk of developing PC compared to patients without VTE. As reviewed by Sohail et al. [19] a decrease in PS level is among the causes of hypercoagulability in PC and hypercoagulability is more common in PC patients than any other gastrointestinal tumors.

Notably, treatment of PC patients with anticoagulant warfarin along with heparin, and, in a separate study, addition of low molecular weight heparin (LMWH) in combination with chemotherapy compared with chemotherapy alone resulted in increased survival benefit in PC patients [19]. In addition to its anti-coagulant activity, PS, the natural anticoagulant product of the PROS1 gene, has multiple coagulation- unrelated effects mediated by TAM (Tyro3, Axl, Mer) receptors [20]. The expression levels of the TAM receptors vary across cell and tissue types and are generally upregulated in tumors [11,21]. As a result, the downstream effects of TAM signaling, such as proliferation and antiinflammatory activity, become dysregulated [22,23]. Shen et al. [24] have extensively discussed the notion of targeting TAM receptors to inhibit cancer progression. However, despite considerable effort, TAM-inhibitory drugs have had minimal success. Recent evidence from Peeters et al. [25] indicates that TAM receptor, MERTK sends a late co-stimulatory signal required for long-lasting anti-tumor CD8+ $\mathrm{T}$ cell responses and the establishment of immunological memory. The pathological complexity of cancer and the global ramifications of TAM-dependent signaling pathways are significant obstacles for use of these drugs because they cause severe side effects and are associated with drug resistance. Hypoxia is a notable feature of the TME and functions as one of the drivers of tumor progression. Hypoxia also promotes the maintenance of tumor-initiating cells. These cells, also known as cancer stem cells or CSCs, oftentimes linked to tumor recurrence in patients [26], metastatic progression, and acquired drug resistance $[27,28]$. One of the mechanisms whereby hypoxia promotes CSC survival is through Notch signaling [29]. A hypoxia-induced transmembrane glycoprotein, Vasorin has been linked to upregulate Notch in CSCs, rescuing Notch from Numb mediated degradation in glioblastoma [7]. PC and other cancers are characterized by a hypoxic niche [6] that stabilizes HIF1 $\alpha$ or HIF2 $\alpha$, which in turn stabilizes Notch [7,30]. HIF1 $\alpha$ induces Notch1 in PC cells [31]. Interestingly, in neural stem cell, PS downregulates Notch1 [32]. However, nothing is known regarding the possible effects of PS on Notch signaling in PC or any other cancers and therefore begs further investigation. In the recent past, there is a lot of focus aimed at combating tumor progression by modulating TME. Hypoxia being an integral component of TME and based on the existing literature, we hypothesize that infusion of PS can modulate TME or can desensitize tumor infiltrating lymphocytes (TIL) or reprogram both TME and TIL to control PC progression (Figure1). Notably, FDA just approved Merck's oral HIF2 $\alpha$ inhibitor, Welireg in USA, a first of its kind for adult patients suffering from Von Hippel-Lindau (VHL) gene associated renal cell carcinoma, pancreatic neuroendocrine tumors or central nervous system hemangioblastomas (https:// www.merck.com/news/fda-approves-mercks-hypoxia-induciblefactor-2-alpha-hif-2\%CE\%B1-inhibitor-welireg-belzutifan-for-thetreatment-of-patients-with-certain-types-of-von-hippel-lindauvhl-disease/) paving way for more active research in this field.

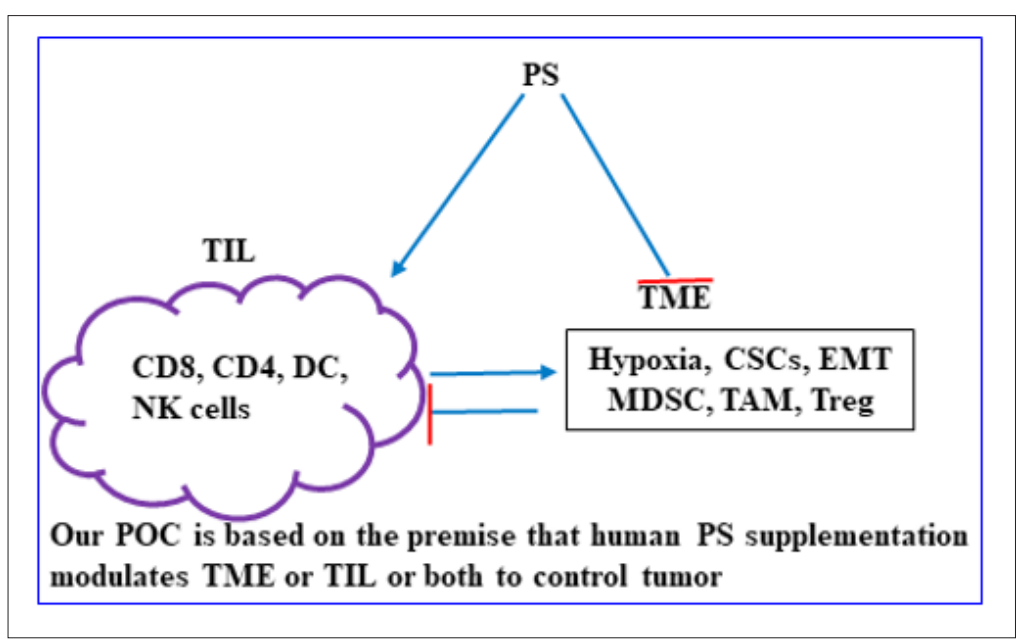

Figure 1:

The blue arrow represents upregulation and and red line indicates downregulation.

\section{Conclusion}

Given PS is easy to manufacture and formulate, and is a natural anticoagulant, it will be interesting to investigate PS efficacy in preclinical studies first. PS supplementation of $2 \mathrm{mg} / \mathrm{kg}$ to attain neural protection in mice in brain injury model was tested without adverse effect of bleeding [33]. However, there is no literature to document PS supplementation to control tumor. If preclinical studies look promising, a rational product development strategy of PS will produce proof of concept for a viable first-in-class investigational product for clinical development. 


\section{References}

1. Carpelan-Holmstrom M, Nordling S, Pukkala E, Sankila R, Luttges J, et al. (2005) Does anyone survive pancreatic ductal adenocarcinoma? A nationwide study re-evaluating the data of the Finnish Cancer Registry. Gut 54(3): 385-387.

2. Waters AM, Der CJ (2018) KRAS: The critical driver and therapeutic target for pancreatic cancer. Cold Spring Harbor Perspectives in Medicine 8(9): a031435.

3. Aglietta M, Barone C, Sawyer MB, Moore MJ, Miller WH, et al. (2014) A phase I dose escalation trial of tremelimumab (CP-675,206) in combination with gemcitabine in chemotherapy-naive patients with metastatic pancreatic cancer. Ann Oncol 25(9): 1750- 1755.

4. Brunet LR, Hagemann T, Andrew G, Mudan S, Marabelle A (2016) Have lessons from past failures brought us closer to the success of immunotherapy in metastatic pancreatic cancer? Oncoimmunology 5(4): e1112942.

5. Satyananda V, Gupta R, Hari DM, Yeh J, Chen KT (2019) Advances in translational research and clinical care in pancreatic cancer: Where are we headed? Gastroenterol Res Pract, 7690528.

6. Erkan M, Kurtoglu M, Kleeff J (2016) The role of hypoxia in pancreatic cancer: a potential therapeutic target? Expert Review of Gastroenterology \& H 10(3): 301-316.

7. Man J, Yu X, Huang H, Zhou W, Xiang C, et al. (2018) Hypoxic induction of vasorin regulates notch1 turnover to maintain glioma stem-like cells. Cell Stem Cell 22(1): 104-118 e106.

8. Koong AC, Mehta VK, Le QT, Fisher GA, Terris DJ, et al. (2000) Pancreatic tumors show high levels of hypoxia. International Journal of Radiation Oncology, Biology, Physics 48(4): 919-922.

9. Tao J, Yang G, Zhou W, Qiu J, Chen G, et al. (2021) Targeting hypoxic tumor microenvironment in pancreatic cancer. Journal of Hematology \& Oncology 14: 14

10. Qin Y, Zhu W, Xu W, Zhang B, Shi S, et al. (2014) LSD1 sustains pancreatic cancer growth via maintaining HIF1alpha-dependent glycolytic process. Cancer Letters 347(2): 225-232.

11. Pilli VS, Datta A, Dorsey A, Liu B, Majumder R (2020) Modulation of protein $S$ and growth arrest specific 6 protein signaling inhibits pancreatic cancer cell survival and proliferation. Oncology Reports 44(4): 1322-1332.

12. Pilli VS, Plautz W, Majumder R (2016) The journey of protein s from an anticoagulant to a signaling molecule. JSM Biochemistry and Molecular Biology 3(1): 1014

13. Dorsey A, Pilli VS, Fried H, Majumder R (2017) Protein S: A multifunctional anticoagulant. Biomed Res Clin Pract 2(5): 15761.

14. Lindahl AK, Odegaard OR, Sandset PM, Harbitz TB (1992) Coagulation inhibition and activation in pancreatic cancer. Changes during progress of disease. Cancer 70(8): 2067-2072.

15. Pilli VS, Datta A, Afreen S, Catalano D, Szabo G, et al. (2018) Hypoxia downregulates protein S expression. Blood 132(4): 452-455.

16. Mandala M, Falanga A, Roila F, EGW (2011) Management of Venous Thromboembolism (VTE) in cancer patients: ESMO Clinical Practice Guidelines. Annals of Oncology 22 (Suppl 6): vi85-92.
17. Hisada Y, Geddings JE, Ay C, Mackman N (2015) Venous thrombosis and cancer: from mouse models to clinical trials. Journal of Thrombosis and Haemostasis 13(8): 1372-1382.

18. Iodice S, Gandini S, Lohr M, Lowenfels AB, Maisonneuve P (2008) Venous thromboembolic events and organ-specific occult cancers: A review and meta-analysis. Journal of Thrombosis and Haemostasis 6(5): 781-788.

19. Sohail MA, Saif MW (2009) Role of anticoagulation in the management of pancreatic cancer. Journal of the Pancreas 10(2): 82-87.

20. Hafizi S, Dahlback B (2006) Gas6 and protein S. Vitamin K-dependent ligands for the Axl receptor tyrosine kinase subfamily. The FEBS Journal 273(23): 5231-5244.

21. Linger RM, Keating AK, Earp HS, Graham DK (2008) TAM receptor tyrosine kinases: biologic functions, signaling, and potential therapeutic targeting in human cancer. Advances in Cancer Research 100: 35-83.

22. Graham DK, Salzberg DB, Kurtzberg J, Sather S, Matsushima GK, et al. (2006) Ectopic expression of the proto-oncogene Mer in pediatric T-cell acute lymphoblastic leukemia. Clinical Cancer Research: An Official Journal of the American Association for Cancer Research 12(9): 26622669.

23. Shankar SL, O'Guin K, Cammer M, McMorris FA, Stitt TN, et al. (2003) The growth arrest-specific gene product Gas6 promotes the survival of human oligodendrocytes via a phosphatidylinositol 3-kinase-dependent pathway. The Journal of Neuroscience 23(10): 4208-4218.

24. Shen Y, Chen X, He J, Liao D, Zu X (2018) Axl inhibitors as novel cancer therapeutic agents. Life sciences 198: 99-111.

25. Peeters MJW, Dulkeviciute D, Draghi A, Ritter C, Rahbech A (2019) MERTK acts as a costimulatory receptor on human CD8(+) T cells. Cancer Immunology Research 7(9): 1472-1484.

26. Pattabiraman DR, Weinberg RA (2014) Tackling the cancer stem cells what challenges do they pose? Nature reviews. Drug Discovery 13(7): 497-512.

27. Yao W, Maitra A (2019) Hear pancreatic cancer stem cells ROR. Cell 177(3): 516-518.

28. Ishiwata T, Matsuda Y, Yoshimura H, Sasaki N, Ishiwata S, et al. (2018) Pancreatic cancer stem cells: Features and detection methods. Pathology Oncology Research 24(4): 797-805.

29. Majumder S, Crabtree JS, Golde TE, Minter LM, Osborne BA, et al. (2021) Targeting Notch in oncology: The path forward. Nature Reviews Drug Discovery 20(2): 125- 144.

30. Kim Y, Lin Q, Zelterman D, Yun Z (2009) Hypoxia-regulated delta-like 1 homologue enhances cancer cell stemness and tumorigenicity. Cancer Research 69(24): 9271-9280.

31. Chen DW, Wang H, Bao YF, Xie K (2018) Notch signaling molecule is involved in the invasion of MiaPaCa2 cells induced by $\mathrm{CoCl} 2$ via regulating epithelialmesenchymal transition. Molecular Medicine Reports 17(4): 4965-4972.

32. Zelentsova K, Talmi Z, Abboud-Jarrous G, Sapir T, Capucha T, et al. (2017) Protein $S$ regulates neural stem cell quiescence and neurogenesis. Stem cells 35(3): 679-693.

33. Liu D, Guo H, Griffin JH, Fernandez JA, Zlokovic BV (2003) Protein S confers neuronal protection during ischemic/hypoxic injury in mice. Circulation 107(13): 1791-1796. 